\title{
Bioactive evaluation and application of different formulations of the natural colorant curcumin (E100) in a hydrophilic matrix (yogurt)
}

Running Title: Different formulations of the natural colorant curcumin (E100)

Heloísa H.S. Almeida ${ }^{1,2,3}$, Lillian Barros ${ }^{1}$, João C.M. Barreira ${ }^{1}$, Ricardo C. Calhelha ${ }^{1}$, Sandrina A. Heleno ${ }^{1,2}$, Claudia Sayer ${ }^{4}$, Cristiane Grella Miranda ${ }^{3}$, Fernanda Vitória Leimann $^{2,3}$, Maria Filomena Barreiro ${ }^{1,2, *}$, Isabel C.F.R. Ferreira ${ }^{1, *}$

${ }^{1}$ Centro de Investigação de Montanha (CIMO), Instituto Politécnico de Bragança, Campus de Santa Apolónia, 5300-253 Bragança, Portugal

${ }^{2}$ Laboratory of Separation and Reaction Engineering - Laboratory of Catalysis and Materials (LSRE-LCM), Polytechnic Institute of Bragança, Campus Santa Apolónia, 5301-857 Bragança, Portugal.

${ }^{3}$ Post-Graduation Program of Food Technology (PPGTA), Federal University of Technology Paraná - UTFPR, Campus Campo Mourão, via Rosalina Maria dos Santos, 1233, CEP 87301899, Caixa Postal: 271, Campo Mourão, PR, Brazil.

${ }^{4}$ Chemical and Food Engineering Department, Federal University of Santa Catarina, CTC, Caixa Postal 476, CEP 88040-970, Florianópolis, SC, Brazil

* Authors to whom correspondences should be addressed (e-mail: iferreira@ipb.pt; telephone +351273303219; fax +351273325405; barreiro@ipb.pt; telephone +351273303089; fax $+351273325405)$.

\section{Abstract}


Curcumin (E100) is a natural colorant that, besides conferring color, has bioactivity, serving as an alternative to some artificial colorants. As a hydrophobic colorant, its modification/compatibilization with the aqueous medium is required to improve stability and enable its application in hydrophilic food matrices. Herein, different formulations of curcumin (curcumin powder: PC, water-dispersible curcumin: DC: and nanoencapsulated curcumin: NC) were evaluated as yogurt colorants. PC showed the strongest bioactivity in all assays $\left(\mathrm{EC}_{50}\right.$ values: $63 \pm 2$ to $7.9 \pm 0.1 \mu \mathrm{g} . \mathrm{mL}^{-1}$; $\mathrm{GI}_{50}$ values: $48 \pm 1$ to $17 \pm 1 \mu \mathrm{g} \cdot \mathrm{mL}^{-1}$ and MIC values: 0.0625 to $0.5 \mathrm{mg} \cdot \mathrm{mL}^{-1}$ ), which might indicate that $\mathrm{DC}$ and $\mathrm{NC}$ reduce the short-term accessibility to curcumin. The tested curcumin formulations produced yogurts with different appearance, specifically associated with their color parameters, besides presenting slight changes in nutritional composition and free sugars and fatty acids profiles. The water compatible formulations (DC and NC) showed advantages over hydrophobic (PC) having a wider industrial utilization.

Keywords: Curcumin; Bioactivity; Nanoencapsulation; Yogurt incorporation. 


\section{Introduction}

Additives exert truly important benefits on the shelf life, microbiological quality and safety of numerous foodstuffs. Not least important is the sensorial attractiveness of these products, namely their scent and color, which justify the great importance of food colorants, particularly because color is often regarded as a quality indicator (Martins, Roriz, Morales, Barros, \& Ferreira, 2016). According to Food and Drug Administration (FDA), a food colorant is "any colorant, pigment or substance which, when added or applied to a food, drug or cosmetic, or to the human body, is capable (alone or through reactions with other substances) of imparting color" (Martins et al., 2016). The main reasons for adding food colorants to foods are: (i) to restore natural color lost during processing and storage; (ii) to reduce batch-to-batch variation of products; (iii) to supplement the intensity of natural colors when these are weak; (iv) to impart color to colorless products; and, (v) to produce acceptable and attractive products for the consumer (Damant, 2011). These additives can be synthesized or extracted from plants or animals (natural sources).

Although being more stable and easier to apply in food products, synthetic and artificial colorants are being gradually replaced by natural alternatives due to the risks they present to human health, such as cumulative effects related to daily intake. However, and despite being highly accepted in the market, it is necessary to develop techniques to optimize the application of these colorants, especially in the cases of natural compounds that were already validated as potential substitutes of artificial molecules. Curcumin (E100), urucum (E160b), lutein (E161b) and other carotenoids, for example, might be suitable natural alternatives to Tartrazine (E102), a widespread food colorant that has been linked to irritability, restlessness and sleep disturbance in children (Carocho, Morales, \& Ferreira, 2015). Curcumin is a hydrophobic yellow-orange polyphenol derived from the rhizome of the herb Curcuma longa L., with effective application in food and pharmaceutical preparations. In food industry, curcumin is added as a stabilizer (in 
jellies) or as a natural colorant, substituting artificial colorants in cheeses, pickles, mustards, cereals, soups, ice creams and yogurts (Mangolim et al., 2014). Besides its coloring capacity, curcumin also possesses antibacterial, anti-proliferative, anti-inflammatory, antioxidant and anti-carcinogenic properties (Mahmood, Zuber, Salman, \& Anjum, 2015).

The major barriers for curcumin's use in food processing industry are its poor water solubility and susceptibility to alkaline conditions, light, oxidation and heat, which also limit its clinical efficacy (Paramera, Konteles, \& Karathanos, 2011). Therefore, numerous technologies have been developed to improve its aqueous solubility, to prevent its degradation and oxidation, and to raise its oral bioavailability (Shin, Kim, \& Park, 2015).

Complementarily, the nano-encapsulation of functional ingredients improves the physical stability of compounds, in and out of the gut, besides enhancing the uptake of components during digestion (Rao \& Khanum, 2015). It is also important that the applied encapsulation technique may increase the water solubility of the target compound, as it was previously verified with the solid dispersion encapsulation technique, which successfully improved the water solubility of hydrophobic molecules such as curcumin (Kaewnopparat, Kaewnopparat, Jangwang, Maneenaun, Chuchome, \& Panichayupakaranant, 2009, Yen, Wu, Tzeng, Lin, \& Ling, 2010, Jithan, Madhavi, Madhavi, \& Prabhakar, 2011, Martins, Pereira, Siqueira, Salomão, \& Freitas, 2013) and lutein (do Prado Silva et al., 2017). In this technique, a mixture of the encapsulating polymer and the bioactive compound to be encapsulated (hydrophobic) are solubilized in a common organic solvent (ethanol or a hydro-alcoholic solution), and then the mixture is subjected to sonication to induce the interaction (hydrogen bonding), between the polymer and encapsulated molecules (Karavas, Ktistis, Xenakis, \& Georgarakis, 2006). Finally, at the end of the process, all the solvents are evaporated (Silva et al., 2016). Polymers with hydrophilic properties are preferred such as polyvinylpyrrolidone (PVP) which is also biocompatible and already used in the food industry. 
Herein, the solid dispersion technique was employed to encapsulate curcumin in PVP, obtaining nanoencapsulated curcumin (NC). The bioactivity of this formulation was compared with a powder formulation containing $65 \%$ of curcumin (PC) and a commercial water-dispersible sample of curcumin (DC). Each assayed formulation was further incorporated in yogurt, which was selected as an hydrophilic model. Afterwards, the color parameters and nutritional value of the prepared yogurts were compared in three consecutive periods ( 0,7 and 15 days), in order to assess their shelf life quality.

\section{Materials and methods}

\subsection{Samples}

Curcumin (from Curcuma longa (Turmeric) powder, $\geq 65 \%$, Sigma-Aldrich, St. Louis, MO, USA), polyvinylpirrolidone (PVP, 40,000 g. $\mathrm{mol}^{-1}$, Sigma-Aldrich, St. Louis, MO, USA), Tween 80 (Dinâmica, Diadema, SP, Brazil) and ethanol (99.5\%, Neon, Suzano, SP, Brazil) were used in nanoparticles preparation. Curcumin itself (designated as curcumin powder, PC), and a commercial water-dispersible formulation (DC) from CHR Hansen (Hoersholm, Denmark) were used for comparison with the nanoencapsulated formulation (NC). The DC sample comprises a curcumin content $4.75-5.25 \%, 75 \%$ of Tween 80 and $19 \%$ of PEG; it corresponds to a viscous liquid with a color ranging from orange to brown.

\subsection{Reagents and standards}

Methanol, dimethyl-sulfoxide (DMSO), petroleum ether, and ethanol were of analytical grade and acquired from Fisher Scientific (Lisbon, Portugal). The fatty acids methyl ester (FAME) reference standard mixture 37 (standard $47885-\mathrm{U}$ ), as also other individual fatty acid isomers, sugar standards, Trolox (6-hydroxy-2,5,7,8-tetramethylchroman-2-carboxylic acid), $\beta$ carotene, dexamethasone, and ellipticine were purchased from Sigma-Aldrich (St. Louis, MO, 
USA), as also acetic acid, phosphate buffered saline (PBS), sulforhodamine B (SRB), trypan blue, and lipopolysaccharide (LPS). Dulbecco's Modified Eagle's Medium (DMEM) and RPMI-1640 medium, fetal bovine serum (FBS) and penicillin/streptomycin solution (100 U/mL and $100 \mathrm{mg} \cdot \mathrm{mL}^{-1}$, respectively) were from Hyclone (Logan, UT, USA). The Griess reagent system was purchased from Promega Corporation (Madison, WI, USA). The culture media Muller Hinton broth (MHB) and Tryptic Soy Broth (TSB) were obtained from Biomerieux (Marcy l'Etoile, France). The colorant $p$-iodonitrotetrazolium chloride (INT) was purchased from Sigma-Aldrich (Spruce Street; St. Louis, MO) and was used as microbial growth indicator. All other chemicals and solvents were of analytical grade and purchased from common suppliers. A Milli-Q water purification system was used for water treatment (TGI Pure Water Systems, Greenville, SC, USA).

\subsection{Nanoencapsulated curcumin by solid dispersion}

\subsubsection{Synthesis procedure}

The nanoencapsulated curcumin, comprised of powder curcumin (65\% pure grade) and the PVP polymer (polyvinylpirrolidone), was prepared by the dissolution in common solvent method (solid dispersion) (Karavas et al., 2006; Miranda et al., 2016). Initially, PVP (100 mg) was dissolved in ethanol $(27.5 \mathrm{~mL})$ under magnetic stirring until obtaining a translucent solution. After that, curcumin $(10 \mathrm{mg})$ and Tween $80(10 \mathrm{mg})$ were added to this solution, remaining in mild stirring for $5 \mathrm{~min}$. The mixture was then sonicated for $15 \mathrm{~min}$ under pulse condition $(30 \mathrm{~s}$ on/10 s off) at $120 \mathrm{~W}$ and using a 1/8' tip (Fisher Scientific, Loughborough, UK) under controlled temperature (thermostatic bath, Quimis, Diadema, SP, Brazil) at $25^{\circ} \mathrm{C}$. Finally, the solvent was evaporated in an air circulation oven (Cienlab, Campinas, SP, Brazil) at $40^{\circ} \mathrm{C}$ for $24 \mathrm{~h}$ and the resulting solid grinded with a pistil and mortar. The theoretical content of curcumin was $8.33 \times 10^{-2} \mathrm{mg} \cdot \mathrm{mg}_{\text {solids }}{ }^{-1}$ (equivalent to $8.3 \% \mathrm{w} / \mathrm{w}$ ). 


\subsubsection{Nanoparticles characterization}

Nanoparticles intensity average diameter (Dz) and polydispersity index (PDI) were determined by Dynamic Light Scattering (DSL, NanoSizer, Malvern Instruments, Malvern, UK) after nanoparticles dispersion in distilled water $(1 \% \mathrm{w} / \mathrm{v})$. The morphology was assessed by Transmission Electron Microscopy (TEM, JEOL JEM- 1011, Peabody, USA). For that, nanoparticles were dispersed in distilled water $(0.1 \% \mathrm{w} / \mathrm{v})$, then dropped in a cooper grid (200 Mesh Tyler) previously covered with collodion film and kept in a desiccator until analysis at $80 \mathrm{kV}$.

For the thermogravimetric analyses (TG 209-F3, Netzsch) approximately $8 \mathrm{mg}$ of each sample was weighed into an alumina crucible and analyzed starting at $20^{\circ} \mathrm{C}$ until $710{ }^{\circ} \mathrm{C}\left(10^{\circ} \mathrm{C} \cdot \mathrm{min}^{-1}\right)$ under nitrogen flow $\left(20 \mathrm{~mL} \cdot \mathrm{min}^{-1}\right)$. Nanoencapsulated curcumin (NC), pure curcumin (PC), the original polymer (PVP), as well as physical mixtures of curcumin and PVP $(4,8,16$ and 50\% $\mathrm{w} / \mathrm{w}$ curcumin) were evaluated. All samples were placed in a vacuum oven at $400 \mathrm{mmHg}$ and $50{ }^{\circ} \mathrm{C}$ for 24 hours before analyses.

Infrared Fourier Transform Spectroscopy (FTIR) spectra were obtained in a MB300 (ABB, Zurich, Switzerland) apparatus in transmittance mode using the following parameters: 32 scans. $\mathrm{min}^{-1}, 16 \mathrm{~cm}^{-1}$ resolution and a scanning range of 4000-550 $\mathrm{cm}^{-1}$. Spectra were obtained with Horizon MB software version 3.4 and normalized to allow comparison. Samples $(1 \% \mathrm{w} / \mathrm{w})$ were finely dispersed in $\mathrm{KBr}$ and pelletized before analyses.

\subsection{Colorants biological activity evaluation}

\subsubsection{Evaluation of the colorants antioxidant activity}

All the colorant samples were dissolved in methanol at a final concentration of $1000 \mu \mathrm{g} \cdot \mathrm{mL}^{-1}$, thereafter diluted to $2 \mu \mathrm{g} \cdot \mathrm{mL}^{-1}$. The results were expressed as $\mathrm{EC}_{50}$, the sample concentration 
providing $50 \%$ of biological activity (DPPH, $\beta$-carotene and TBARS assays) or 0.5 of absorbance (reducing power assay). Trolox was used as the standard positive control in all assays that were carried out in triplicate (Calhelha, Peixoto, Vilas Boas, Queiroz, \& Ferreira, 2014). 2,2-Diphenyl-1-picrylhydrazyl (DPPH) radical scavenging activity, reducing power, inhibition of $\beta$-carotene bleaching and the lipid peroxidation inhibition in porcine brain homogenates were evaluated following a previously described procedure (Calhelha, Peixoto, Vilas Boas, Queiroz, \& Ferreira, 2014).

\subsubsection{Evaluation of the colorants anti-inflammatory activity}

PC was dissolved in a dimethyl-sulfoxide:water mixture (DMSO:water, 50:50, v/v), while NC and DC were dissolved in water at a stock concentration of $8 \mathrm{mg} \cdot \mathrm{mL}^{-1}$, being further diluted to different concentrations (400-6.25 $\mu \mathrm{g} \cdot \mathrm{mL}^{-1}$ ). Results were expressed as percent inhibition of NO production compared to the negative control (100\%) and $\mathrm{EC}_{50}$ values (concentration of the sample providing 50\% inhibition of NO production) were also estimated. Dexamethasone (50 $\mu \mathrm{M})$ was used as the positive control in the experiment.

A method previously described by Correa et al. (2015) was carried out. The mouse macrophages cell line (RAW 264.7) was cultured using a DMEM supplemented with $10 \%$ heat-inactivated fetal bovine serum and glutamine, at $37^{\circ} \mathrm{C}$ under $5 \% \mathrm{CO}_{2}$, in humidified air. The cell density was $5 \times 10^{5}$ cells. $\mathrm{mL}^{-1}$ and the proportion of dead cells was less than $1 \%$ according to trypan blue colorant exclusions tests. The cells were plated in 96-well plates, each well containing 150.000 cells, and the plate left in the incubation chamber overnight. Then the cells were treated with different concentrations of each sample for $1 \mathrm{~h}$ and further stimulated with lipopolysaccharide (LPS) $\left(1 \mu \mathrm{g} . \mathrm{mL}^{-1}\right)$ for $18 \mathrm{~h}$. The effect of the samples, tested in the absence of LPS, was also evaluated to check if they induced changes in basal nitric oxide (NO) levels. To determine NO concentration, a Griess reagent kit containing sulphanilamide, N-(1-naphthyl) 
ethylenediamine dihydrochloride (NED) and a nitric acid solution was used. A portion of 100 $\mu \mathrm{L}$ of the cell culture supernatant was transferred to a duplicate plate by mixing the sulphanilamide with the NED solution, respectively, and allowed to stand for 5 to $10 \mathrm{~min}$ each at room temperature. The nitrite production was determined by measuring the absorbance at $515 \mathrm{~nm}$ in the mentioned above plate reader and compared to the standard calibration curve.

\subsubsection{Evaluation of the colorants cytotoxicity}

PC, NC and DC were dissolved, as previously mentioned, for the anti-inflammatory activity assay and the working concentration ranged from $400-6.25 \mu \mathrm{g} \cdot \mathrm{mL}^{-1}$. Ellipcitine was used as positive control and the results were expressed as $\mathrm{GI}_{50}$ values (sample concentration that inhibited $50 \%$ of the net cell growth) in $\mu \mathrm{g} \cdot \mathrm{mL}^{-1}$.

The cytotoxicity was evaluated by sulforhodamine B (SRB) assay in four human tumor cell cultures: MCF-7 (breast adenocarcinoma), NCI-H460 (lung carcinoma), HepG2 (hepatocellular carcinoma) and HeLa (cervical carcinoma). Cells were routinely maintained as adherent cell culture in RPMI-1640 containing 10\% heat-inactivated FBS and $2 \mathrm{mM}$ glutamine at $37{ }^{\circ} \mathrm{C}$ in a humidified air incubator containing $5 \% \mathrm{CO}_{2}$. Each cell line was plated at an appropriate density (Barros et al., 2013).

A non-tumor cell line, prepared from a freshly harvested porcine liver and designated as PLP2, was also evaluated. Briefly, the liver tissue was rinsed in Hank's balanced salt solution containing $100 \mathrm{U} \cdot \mathrm{mL}^{-1}$ penicillin plus $100 \mu \mathrm{g} \cdot \mathrm{mL}^{-1}$ streptomycin, and was divided into $1 \times 1 \mathrm{~mm}^{3}$ explants. Some of them were placed into $25 \mathrm{~cm}^{2}$ tissue flask containing DMEM medium (supplemented with $10 \%$ fetal bovine serum, $2 \mathrm{mM}$ non-essential amino acids and $100 \mathrm{U}_{\mathrm{mL}}^{-1}$ penicillin, $100 \mu \mathrm{g} \cdot \mathrm{mL}^{-1}$ streptomycin) and incubated at $37^{\circ} \mathrm{C}$ under a humidified atmosphere with $5 \% \mathrm{CO}_{2}$. Phase contrast microscope was used for direct monitoring of the cell cultivation every 2 to 3 days. 


\subsubsection{Evaluation of the colorants antibacterial activity}

For this methodology four Gram positive (methicillin-resistant Staphylococcus aureus MRSA), methicillin-sensitive Staphylococcus aureus - MSSA, Enterococcus faecalis and Listeria monocytogenes) and six Gram negative (Escherichia coli, Escherichia coli producing $\beta$-lactamase, Klebsiella pneumoniae, Klebsiella pneumoniae producing $\beta$-lactamase, Morganella morganii and Pseudomonas aeruginosa) bacteria were tested. Initially, $48 \mathrm{~h}$ prior to analysis, the bacteria were repackaged to fresh medium followed by incubation at $37{ }^{\circ} \mathrm{C}$ for $24 \mathrm{~h}$ and subsequent repackaging to fresh medium and incubation at $37{ }^{\circ} \mathrm{C}$ for further $24 \mathrm{~h}$, ensuring that the bacteria would be in the exponential phase of growth. Curcumin powder was prepared by dissolving $10 \mathrm{mg}$ in $1 \mathrm{~mL}$ of $50 \%$ DMSO. Afterwards, one additional $\mathrm{mL}$ of water with $25 \%$ of DMSO was added and further diluted, with medium and bacteria, by 5 times (1 mg.mL $\mathrm{mL}^{-1}, 5 \% \mathrm{DMSO}$ ); the other samples (DC and $\mathrm{NC}$ ) were dissolved in the culture medium (1 mg.mL $\mathrm{m}^{-1}$ ). The determination of the minimal inhibitory concentration (MIC) was performed by the microdilution method with rapid INT colorimetric assay (Svobodova et al., 2017).

\subsection{Incorporation of the colorant in yogurts}

\subsubsection{Yogurt preparation}

The natural (prepared with milk obtained from organic farming) yogurts were purchased at a local supermarket in Bragança, Portugal. They comprise the following nutritional information (per $100 \mathrm{~g}$ of the product): energy: $316 \mathrm{~kJ}$ (74 kcal); lipids: $3.8 \mathrm{~g}$, saturated: $2.4 \mathrm{~g}$; carbohydrates: $4.7 \mathrm{~g}$, of which sugars: $4.7 \mathrm{~g}$; proteins: $5 \mathrm{~g}$; salt: $0.15 \mathrm{~g}$. The incorporation of the colorant formulations was performed by adding directly the colorant until a pre-defined color parameter ( $b^{*}$ value form CIE Lab colors) was achieved, leading to different used quantities as expected from the different hydrophilic character of curcumin formulations (50 mg of PC, 7 
$\mathrm{mg}$ of $\mathrm{DC}$ and $5.5 \mathrm{mg}$ of $\mathrm{NC}$ in $70 \mathrm{~g}$ of yogurt). In any case, the used quantities respect the limits established by the legislation and acceptable daily dose ( $3 \mathrm{mg}$ per $\mathrm{kg}$ body weight per day, EFSA, 2014).

Four groups of yogurt samples were prepared: i) control yogurt (without any incorporation of colorants - YC); ii) yogurts incorporated with the curcumin powder (YPC); iii) yogurts incorporated with the commercial water-dispersible formulation (YDC); and iv) yogurts incorporated with the produced nanoparticles formulation (YNC). All samples were prepared in triplicate to evaluate three different storage times: immediately after preparation, after 3 and 7 days of storage at $4{ }^{\circ} \mathrm{C}$.

\subsubsection{Color evaluation}

The monitoring of the colorant ability and its uniformity in the final product was performed using a colorimeter (model CR-400, Konica Minolta Sensing Inc., Japan). The illuminate C and a diaphragm aperture of $8 \mathrm{~mm}$ were chosen after calibration against a standard white tile. The CIE $L^{*}$ (lightness), $a^{*}$ (redness), $b^{*}$ (yellowness) color space values were registered using the data software "Spectra Magic Nx" (version CM-S100W 2.03.0006) (Fernandes, Antonio, Barreira, Oliveira, Martins, \& Ferreira, 2012).

\subsubsection{Evaluation of the nutritional composition and curcumin quantification along storage} time

Lyophilized samples were analyzed for ash, protein, fat and carbohydrate content, following official food analysis procedures (AOAC, 2016). Ash content was determined by incineration of a given sample mass at $600{ }^{\circ} \mathrm{C}$; protein $(\mathrm{N} \times 6.38)$ was determined by the macro-Kjeldahl method using equipment (model Pro-nitro M Kjeldhal Steam Distillation System, Barcelona, Spain); fat was quantified after Soxhlet extraction with petroleum ether; total carbohydrates 
were obtained by difference. Energy was calculated from the equation: $E=4 \times(P+C)+9 \times F$, where E represents the energy ( $\left.\mathrm{kcal} 100 \mathrm{~g}^{-1}\right), \mathrm{P}$ the amount of protein, $\mathrm{C}$ the amount of carbohydrates and $\mathrm{F}$ the amount of fat, all expressed in $\mathrm{g} .100 \mathrm{~g}^{-1}$ of lyophilized sample.

Fatty acids were determined, after Soxhlet extraction, by gas chromatography coupled to flame ionization detector (GC-FID) and identified by comparison with standards (standard 47885, Sigma-Aldrich, St. Louis, Missouri, USA) and expressed as relative percentages of each fatty acid (Barros et al., 2013).

Free sugars were determined in defatted samples by HPLC coupled to a refraction index (RI) detector (Barros et al., 2013), identified by comparison with standards, and further quantified (g.100 g-1 of yogurt) using an internal standard (melezitose).

The toxicity was evaluated using a primary cell culture prepared from a freshly harvested porcine liver (PLP2) as mentioned above (section 2.4.3).

\subsection{Statistical analysis}

All statistical tests (SPSS Statistics for Windows version 22.0, IBM Corp., Armonk, NY, USA) were performed considering a 5\% significance level. Data (with the exception of MIC values) were expressed as mean \pm standard deviation (the number of significant numbers was maintained according to the standard deviation magnitude).

A 2-way analysis of variance (ANOVA) with type III sums of squares of the general linear model (GLM) procedure was applied to compare different yogurt formulations (YF) and storage times (ST). In addition to their individual effect, YF and ST were also evaluated for possible interactions. If no significant interaction was found, the means of the corresponding parameter were classified using Tukey's multiple comparison test, after evaluating the equality of variances (Levene's test). When YF and ST reveal a significant interaction, differences were compared in the estimated marginal means plots (data not provided). 
Furthermore, a linear discriminant analysis (LDA) was used to identify the parameters with the highest changes in result of YF and ST effects. The stepwise technique was applied, based on the Wilks' $K$ test for variable selection ( $F$ to enter: $3.84 ; F$ to be removed: 2.71 ). Only variables with a statistically significant classification performance $(p<0.050)$ were selected by the discriminant models. A leaving-one-out cross validation procedure was carried out to assess the model performance.

\section{Results and discussion}

\subsection{Characterization of solid dispersion nanoencapsulated curcumin}

PVP-curcumin nanoparticles were successfully synthesized by the solid dispersion technique, as it can be observed in the TEM image (Figure 1A1). The prepared particles presented a monomodal and narrow size distribution $(\mathrm{PDI}=0.050 \pm 0.009)$ with diameters ranging from 220 to $712 \mathrm{~nm}$, and an intensity average size (Dz) equal to $377 \pm 15 \mathrm{~nm}$ (Figure 1A2). Previous results demonstrated that $14 \%$ of the added curcumin may become colloidally stable when produced under the same experimental conditions (de Almeida et al, 2018).

The thermogravimetric results of different prepared formulations (curcumin, PVP, curcuminloaded nanoparticles and physical PVP-curcumin mixtures at curcumin concentration of 4\% $\mathrm{wt}$, $8 \% \mathrm{wt}, 16 \% \mathrm{wt}$ and $50 \% \mathrm{wt}$ ), as well as their FTIR spectra (among physical mixtures, only the $8 \%$ curcumin concentration is shown) are presented in Figure 1B.

The thermal degradation of curcumin was located between 200 and $450{ }^{\circ} \mathrm{C}$ (maximum mass loss at $\left.333{ }^{\circ} \mathrm{C}\right)$. The thermograms of the physically mixed PVP-curcumin approached that of pure curcumin as the curcumin concentration increases, meaning that such systems behaved as truly physical mixtures. This was also evident when analyzing the loss of absorbed water at temperatures near $100{ }^{\circ} \mathrm{C}$, behavior supported by the hydrophobic and hydrophilic nature of curcumin and PVP, respectively. As highlighted in Figure 1B2, only a small mass loss was 
detected for the nanoparticles at $100{ }^{\circ} \mathrm{C}$, while a sharp decrease was detected for the physical mixture mimicking the same curcumin concentration $(8.3 \% \mathrm{wt})$. Also, and as expected, no water loss was observed for pure curcumin due to its hydrophobic character. The observed difference between nanoparticles and the corresponding physical mixtures may be explained by the binding between PVP carbonyls and curcumin hydroxyl groups, leading to a decrease in the interaction ability of water towards PVP, which is a strong indication of an efficient curcumin encapsulation, in line with the results reported for PVP-encapsulated lutein (do Prado Silva et al., 2017). The FTIR spectrum of curcumin exhibited the characteristic absorption bands (Paramera, Konteles, \& Karathanos, 2011; Silva-Buzanello et al., 2016; Yallapu, Jaggi, \& Chauhan, 2010) at $1024 \mathrm{~cm}^{-1}$ (C-O groups), $1503 \mathrm{~cm}^{-1}(\mathrm{C}=\mathrm{O}$ and $\mathrm{C}=\mathrm{C}$ groups $)$ and $963 \mathrm{~cm}^{-}$ ${ }^{1}$ (aromatic $\left.\mathrm{C}-\mathrm{H}\right)$, as well as the absorption band relative to the stretching vibration of curcumin hydroxyls $\left(3510 \mathrm{~cm}^{-1}\right)$. Despite being noticeable for the physical mixture of PVP and curcumin, these bands could not be visualized in the obtained nanoparticles spectra, as exemplified with the $3510 \mathrm{~cm}^{-1}$ band in Figure 1B4, indicating that curcumin should be located inside the PVP matrix. In fact, this type of attenuation effect is often considered as an evidence of an efficient encapsulation (Gangurde, Kundaikar, Javeer, Jaiswar, Degani, \& Amin, 2015; Silva-Buzanello et al., 2016; Yallapu et al., 2010).

\subsection{Evaluation of bioactive properties of curcumin samples}

The results obtained in the evaluation of bioactive properties of different curcumin formulations are given in Table 1. Regarding antioxidant activity, PC showed the minimal $\mathrm{EC}_{50}$ values (maximum antioxidant activity) among all the performed tests, while DC gave the weakest activity, despite its higher proximity to NC values. In fact, the results for PC (curcumin with $65 \%$ purity) were remarkable, considering that its $\mathrm{EC}_{50}$ values were similar (inclusively better in the case of TBARS formation inhibition) to those obtained with the positive control (Trolox). 
The main reason explaining these results might attributed to a reduction in the short-term accessibility of curcumin in the case of DC and NC. However, this should not be seen as a drawback, as it might indicate that both DC and NC may be used as protected delivery strategies for curcumin formulations. The positive effects of curcumin in reducing lipid oxidation were previously reported in stored milk incorporated with a curcumin nanoemulsion (Joung, Choi, Kim, Park, Park, \& Shin, 2016). Likewise, curcumin nanoencapsulated in $\alpha$-lactalbumin was also described for its high antioxidant activity (Yi, Fan, Zhang, Wen, Zhao, \& Lu, 2016).

Besides the antioxidant activity, curcumin is also known for its anti-inflammatory properties, having the potential to alleviate inflammatory responses through a variety of mechanisms, including inhibition of pro-inflammatory cytokines, adhesion and infiltration of macrophages, and modulation of immunocytochemical activity (Jiang et al., 2017). According to Svobodova et al. (2017), NO is a pleiotropic mediator produced at inflammatory sites and used to determine the anti-inflammatory activity in LPS (lipopolysaccharide) stimulated RAW 264.7 macrophages. Herein, the anti-inflammatory activity of the different tested curcumin formulations followed the same trend verified for antioxidant activity: $\mathrm{PC}>>\mathrm{NC}>\mathrm{DC}$, indicating a higher bioactive potential for PC, once again mainly attributable to the short-term accessibility to curcumin in the case of NC and DC.

Curcumin has also been reported to inhibit tumor growth in vitro and in vivo, suppress cell proliferation in several cell lines and inhibit tumorigenesis (Fang, Fang, Gou, \& Cheng, 2013). In the present study, PC, NC and DC showed to be cytotoxic against the assayed tumor cell lines, particularly against MCF-7 (GI 50 values: 29 to $\left.47 \mu \mathrm{g} \cdot \mathrm{mL}^{-1}\right)$. In line with the previously observed bioactivity indicators, PC was the most active formulation. However, the studied samples also demonstrated toxicity against non-tumor cells (PLP-2), despite the higher GI50 values (89 to $235 \mu \mathrm{g} \cdot \mathrm{mL}^{-1}$ ). 
Curcumin was previously characterized as having antimicrobial activity, mainly due to its methoxyl and hydroxyl groups, particularly against Bacillus cereus, Bacillus subtilis, Staphylococcus aureus, Staphylococcus mutans, Staphylococcus epidermidis, Escherichia coli, Pseudomonas aeruginosa, Yersinia enterocolitica and Shigella dysenteriae (Silva, 2016). Besides the isolated compound, the aqueous extracts from Curcuma longa rhizome also showed antibacterial activity (MIC: 4 to $16 \mathrm{mg} \cdot \mathrm{mL}^{-1}$; $\mathrm{MBC}$ : 16 to $32 \mathrm{mg} \cdot \mathrm{mL}^{-1}$ ), particularly against species such as Staphylococcus epidermidis, Staphylococcus aureus, Klebsiella pneumoniae and Escherichia coli (Moghadamtousi, Kadir, Hassandarvish, Tajik, Abukakar, \& Zandi, 2014). Accordingly, the antibacterial activity of the curcumin formulations assayed herein was evaluated in Gram positive (MRSA, MSSA, Enterococcus faecalis and Listeria monocytogenes) and Gram negative (Escherichia coli, Escherichia coli ESBL, Klebsiella pneumoniae, Klebsiella pneumoniae ESBL, Morganella morganii and Pseudomonas aeruginosa) bacteria. PC was, again, the most active formulation, but, and as it can be observed in Table 1 all formulations presented antibacterial activity (except DC against Pseudomonas aeruginosa and Morganella morganii). In general, Gram positive bacteria were more sensitive, particularly to $\mathrm{PC}\left(\mathrm{MIC}=0.0625 \mathrm{mg} \cdot \mathrm{mL}^{-1}\right)$.

\subsection{Nutritional value, color parameters and free sugars/fatty acid profile}

The different curcumin formulations were incorporated in yogurts to evaluate their potential capacity in preventing changes in macronutrients, energy, free sugars, color parameters and fatty acids. All evaluated parameters were compared regarding YF (YC, YDC, YNC and YPC) and ST (0, 3 and 7 days). When two factors contribute to the variability of results, their significant interaction is common. Accordingly, in addition to the significance of each individual factor, their interaction $(\mathrm{YF} \times \mathrm{ST})$ was also studied. In all cases where a significant $(p<0.050)$ interaction was found, hampering the statistical classification of the results, 
differences induced by each factor (when significant) were compared according to the EMM plots.

Starting with the nutritional composition, and as it might be concluded from Table 2, the interaction was significant in all cases except fat content, indicating that the effect of ST over water, protein, carbohydrates, lactose, glucose and energy was not the same for all YF (and vice versa). Water was clearly the main component in yogurt (83 to $84 \mathrm{~g} .100 \mathrm{~g}^{-1}$ ), while carbohydrates and proteins varied between 5.9-6.2 g. $100 \mathrm{~g}^{-1}$ and 5.1-5.4 g. $100 \mathrm{~g}^{-1}$, respectively. Fat content, in turn, presented values around 3.5-3.8 g.100 g-1. In general, the detected profile is in agreement with the information provided in the label of $\mathrm{YC}$ (vide 2.5.1.). Regarding the effects of YF and ST, and despite their significance in most occasions (except for ST in fat content), it might be generally concluded that only minor differences were observed for both factors. A similar result was also obtained for galactose and lactose, which showed similar values independently of YF and ST. Nevertheless, according to the EMM plots, it was possible to obtain some general tendencies: water and fat tended to decrease after 7 days of storage, while ash and energy tended to be slightly lower in YC and YPC, respectively.

Regarding color parameters (Table 3), the interaction among factors was also significant in all cases. Either way, the effect of YF was significant $(p<0.050)$ for $L^{*}$ (lower in YPC), $a^{*}$ (lower in YDC and YNC) and $b^{*}$ (lower in YC), while ST caused only significant differences in $a^{*}$ (lower in non-stored yogurts), which might be considered as an initial indicator of the color maintenance along time. To the naked eye (Figure A1, supplementary material), NC and DC, with similar yellow coloration, could be interesting solutions to produce banana or pineapple yogurt, while PC, closer to orange color, can be considered for mango, peach or papaya yogurt. Nevertheless, due to the poor solubility of PC in water, a higher amount of pigment was added in comparison with the other formulations. Furthermore, the color obtained in this yogurt was 
not homogeneous and, therefore, it is not a suitable solution to be used at industrial level, justifying the search for improved formulations such as DC or NC.

In what concerns the main (relative percentage $>1 \%$, Table 4) fatty acids, the effect of YF and ST was also significant in most cases (except C18:0 for YF and C18:1n9c, SFA and MUFA for $\mathrm{ST})$, as their interaction. The most abundant fatty acids were palmitic acid (C16:0), with values around $35 \%$, and oleic acid $(\mathrm{C} 18: \ln 9 \mathrm{c})$, which corresponded to approximately $20 \%$ of total fatty acids. In general, the statistically significant differences corresponded to small variations in the percentages of fatty acids, indicating their stability, independently of the incorporated curcumin formulation.

The prepared yogurts did not reveal cytotoxicity up to the maximum tested concentration (400 $\left.\mu \mathrm{g} \cdot \mathrm{mL}^{-1}\right)$.

\subsection{Linear Discriminant Analysis}

In the previous sections, it was generally concluded that the differences observed for each yogurt parameter did not seem to be relevant (despite statistically significant). However, it would be relevant to verify if, when analyzed all together, the assayed parameters presented enough differences to discriminate each assayed ST or YF.

In the case of ST effect (Figure 2a), the two defined discriminant functions included 100.0\% (first function: 54.6\%; second function: 48.4\%) of the observed variance. Among the 22 analyzed variables (parameters), 12 were selected as having discriminant effect: water, protein, ash, galactose, lactose, energy, $b^{*}, \mathrm{C} 6: 0, \mathrm{C} 12: 0, \mathrm{C} 14: 0, \mathrm{C} 16: 0$ and PUFA. Among the selected functions, water and protein were the most correlated with function 1. As it might be observed, this function separated markers corresponding to non-stored yogurts and those stored during 7 days, certainly due to the higher water and lower protein contents in non-stored samples. 
Function 2, in turn, was specifically effective to separate "15 days" group markers. The highest correlations among variables and function 2 were obtained for $\mathrm{C} 16: 0$ and C14:0, both presenting lower percentages after 15 days of storage.

Concerning YF effect (Figure 2b), the three defined functions also included $100 \%$ of the observed variance, but in this case with a much higher relevance for function $1(97.0 \%)$. The selected variables were water, ash, energy, C6:0, C10:0, C12:0, C14:0, C18:0, C18:1n9c, SFA and MUFA. The YF with most dissimilarities, according to function 1, were YC and YPC, mostly for their differences in $b^{*}$, which was among the variables with highest correlation with function 1. Function 2, on the other hand, was more correlated with $a^{*}$ and $L^{*}$, thereby validating color parameters as those with the highest differences, while differences in nutritional composition, free sugars and fatty acids profiles were less relevant.

In terms of model performance, the classification was $100 \%$ accurate for both factors (ST and YF) for originally grouped and cross-validated grouped cases.

\section{Conclusion}

This study was specifically designed to compare different formulations of natural curcumin (E100) and their incorporation into yogurts. Before incorporation, all curcumin formulations were evaluated for their bioactivity, having been verified that PC has the highest antioxidant, anti-inflammatory, cytotoxic and antibacterial activity. This might indicate that DC and NC represent effective ways of protecting curcumin, by making it less available in short-term. When incorporated in yogurts, curcumin allowed to achieve an appealing appearance (maintained throughout storage time), without causing relevant changes in the nutritional composition and fatty acids profiles. Despite the similarities among the yogurts incorporated with curcumin, the LDA outputs proved that each yogurt formulation, i.e. yogurts incorporating different curcumin formulations, presented specific changes in nutritional composition, free 
sugars, color parameters and fatty acids. Moreover, the advantages of using modified curcumin formulations (DC and NC) over PC was evident in terms of the achieved color homogeneity, even with much smaller added quantities. The development of new water compatible formulations from hydrophobic colorants, such as curcumin, might represent an advance in bringing these natural solutions to a wider industrial utilization, with unquestionable benefits for consumers.

\section{Acknowledgments}

Authors are grateful to the Foundation for Science and Technology (FCT, Portugal) and FEDER under Programme PT2020 for financial support to CIMO (UID/AGR/00690/2013), L. Barros, João C.M. Barreira and R. Calhelha contracts and Sandrina A. Heleno (SFRH/BPD/101413/2014) grant; to the project POCI-01-0145-FEDER-006984 - Associate Laboratory LSRE-LCM funded by FEDER through COMPETE2020 - Programa Operacional Competitividade e Internacionalização (POCI) - and by national funds through FCT. This work was also funded by the European Structural and Investment Funds (FEEI) through the Regional Operational Program North 2020, within the scope of Project NORTE-01-0145-FEDER023289: DeCodE, project Mobilizador ValorNatural ${ }^{\circledR}$ and project NORTE-01-0145-FEDER000006.

\section{References}

A.O.A.C. (2016). Official methods of analysis of AOAC international. AOAC international, $20^{\mathrm{a}}$ ed.

Barros, L., Pereira, E., Calhelha, R., Dueñas, M., Carvalho, A., Buelga, C. \& Ferreira, I. C.F.R. (2013). Bioactivity and chemical characterization in hydrophilic and lipophilic compounds of Chenopodium ambrosioides L. Journal of functional foods, 5, 1732-1740. 
Calhelha, R. C., Peixoto, D., Vilas Boas, M., Queiroz M. -J. R. P. \& Ferreira I. C. F. R. (2014). Antioxidant activity of aminodiarylamines in the thieno[3,2-b]pyridine series: radical scavenging activity, lipid peroxidation inhibition and redox profile. Journal of Enzyme Inhibition and Medicinal Chemistry, 29, 311-316.

Carocho, M., Morales, P. \& Ferreira, I. C. F. R. (2015). Natural Food Additives: Quo Vadis?. Trends in Food Science \& Technology, 45, 284-295.

Correa, R. C. G., Souza, A. H. P., Calhelha, R. C., Barros, L., Glamoclija, J., Sokovic, M., Peralta, R. M., Bracht, A. \& Ferreira, I. C. F. R. (2015). Bioactive formulations prepared from fruiting bodies and submerged culture mycelia of the Brazilian edible mushroom Pleurotus ostreatoroseus Singer. Food \& Function, 6, 2155-2164.

Damant, A. P. (2011). Food Colourants - Chapter 8. Food Standards Agency, Woodhead Publishing Limited, 252-305.

de Almeida, M., da Rocha, B. A., Francisco, C. R. L., Miranda, C. G., de Freitas Santos, P. D., de Araújo, P. H. H., Sayer, C., Leimann, F. V., Gonçalves, O. H., Bersani-Amado, C. A. (2018). Evaluation of the in vivo acute antiinflammatory response of curcumin-loaded nanoparticles. Food \& Function, 9, 440-449.

do Prado Silva, J. T., Geiss, J. M. T., Oliveira, S. M., Brum, E. da S., Sagae, S. C., Becker, D., Gonçalves, O. H. (2017). Nanoencapsulation of lutein and its effect on mice's declarative memory. Materials Science and Engineering: C, 76, 1005-1011.

EFSA. (2014). Refined exposure assessment for curcumin (E 100), European Food Safety Authority (EFSA), Parma, Italy.

Fang, X., Fang, L., Gou, S. \& Cheng L. (2013). Design and synthesis of dimethylaminomethylsubstituted curcumin derivatives/analogues: Potent antitumor and antioxidant activity, improved stability and aqueous solubility compared with curcumin. Bioorganic \& Medicinal Chemistru Letters, 23, 1297-1301. 
Fernandes, A., Antonio, A. L., Barreira, J. M. C, Oliveira, B. B. P. P., Martins, A. \& Ferreira, I. C. F. R. (2012). Effects of gamma irradiation on physical parameters of Lactarius deliciosus wild edible mushrooms. Postharvest Biology and Technology, 74, 79-84.

Gangurde, A. B., Kundaikar, H. S., Javeer, S. D., Jaiswar, D. R., Degani, M. S., \& Amin, P. D. (2015). Enhanced solubility and dissolution of curcumin by a hydrophilic polymer solid dispersion and its insilico molecular modeling studies. Journal of Drug Delivery Science and Technology, 29, 226-237.

Jiang, S., Han, J., Li, T., Xin, Z., Ma, Z., Di, W., Hu, W., Gong, B., Di, S., Wang, D. \& Yang, Y. (2017). Curcumin as a potential protective compound against cardiac diseases. Pharmacological Research, 119, 373-383.

Jithan, A., Madhavi, K., Madhavi, M., \& Prabhakar, K. (2011). Preparation and characterization of albumin nanoparticles encapsulating curcumin intended for the treatment of breast cancer. International Journal of Pharmaceutical Investigation, 1, 119-125.

Joung, H., Choi, M., Kim, T., Park, S., Park, H. \& Shin, G. (2016). Development of food-grade curcumin nanoemulsion and its potential application to food beverage system: antioxidant property and in vitro digestion. Journal of Food Science, 81, 745-753.

Kaewnopparat, N., Kaewnopparat, S., Jangwang, A., Maneenaun, D., Chuchome, T., \& Panichayupakaranant, P. (2009). Increased Solubility, Dissolution and Physicochemical Studies of Curcumin-Polyvinylpyrrolidone K- Solid Dispersions. International Scholarly and Scientific Research and Innovation, 3, 210-215.

Karavas, E., Ktistis, G., Xenakis, A. \& Georgarakis, E. (2006). Effect of hydrogen bonding interactions on the release mechanism of felodipine from nanodispersions with polyvinylpyrrolidone. European Journal of Pharmaceutics and Biopharmaceutics, 63, 103-114.

Lestari, M. \& Indrayanto, G. (2014) Chapter three - Curcumin. Profiles of Drugs Substances, 
Excipients and Related Methodology, 39, 113-204.

Mahmood, K., Zuber, M., Salman, M., \& Anjum, M. (2015). Recent developments in curcumin and curcumin based polymeric materials for biomedical applications: A review. International Journal of Biological Macromolecules, 81, 877-890.

Mangolim, C., Moriwaki, C., Nogueira, A., Sato, F., Baesso, M., Neto, A. \& Matioli, G. (2014). Curcumin-b-cyclodextrin inclusion complex: Stability, solubility, characterisation by FTIR, FT-Raman, X-ray diffraction and photoacoustic spectroscopy, and food application. Food Chemistry, 153, 361-310.

Martins, N., Roriz, C., Morales, P., Barros, L. \& Ferreira, I. C. F. R. (2016). Food colorants: challenges, opportunities and current desires of agroindustries to enture consumer expectations and regulatory practices. Trends in Food Science \& Technology, 52, 1-15.

Martins, R. M., Pereira, S. V., Siqueira, S., Salomão, W. F., \& Freitas, L. A. P. (2013). Curcuminoid content and antioxidant activity in spray dried microparticles containing turmeric extract. Food Research International, 50, 657-663.

Miranda, C., Silva, J., Becker, D., Guerra, G., Leiman, F., Golçalves, O. \& Ineu, R. (2016). Characterization of lutein nanoparticles in PVP and effects on AChE enzyme activity in vitro. Federal Technological University of Paraná, Brazil.

Moghadamtousi, S., Kadir, H., Hassandarvish, P., Tajik, H., Abukakar, S. \& Zandi, K. (2014). A review on antibacterial, antiviral and antifungal activity of curcumin. BioMed Research Interational, 2014, 1-12.

Paramera, E. I., Konteles, S. J., \& Karathanos, V. T. (2011). Microencapsulation of curcumin in cells of Saccharomyces cerevisiae. Food Chemistry, 125, 892-902.

Rao, P. \& Khanum, H. A green chemistry approach for nanoencapsulation of bioactive compound - Curcumin (2015). LWT - Food Science and Technology, 65, 695-702.

Shin, G. H., Kim, J. T. \& Park, H. J. Recent developments in nanoformulations of lipophilic 
funtional foods (2015). Trends in Food Science \& Technology, 46, 144-157.

Silva, A. (2016). Evaluation of the antimicrobial activity of curcumin nanoparticles and application of microcrystals of curcumin in minimally processed carrots. (Master's thesis). Federal Technological University of Paraná, Brazil.

Silva-Buzanello, R. A., Souza, M. F. De, Oliveira, D. A. De, Bona, E., Leimann, F. V., Filho, L. C., Gonçalves, O. H. (2016). Preparation of curcumin-loaded nanoparticles and determination of the antioxidant potential of curcumin after encapsulation. Polimeros, 26, $207-214$.

Svobodova, B., Barros, L., Calhelha, R. C., Heleno, S. A., Alves, M., Walcott, S., Bittova, M., Kuban, V. \& Ferreira, I. C. F. R. (2017). Bioactive properties and phenolic profile of Momordica charantia L.medicinal plant growing wild in Trinidad and Tobago. Industrial Crops and Products, 95, 365-373.

Yallapu, M. M., Jaggi, M., \& Chauhan, S. C. (2010). $\beta$-Cyclodextrin-curcumin self-assembly enhances curcumin delivery in prostate cancer cells. Colloids and Surfaces B: Biointerfaces, 79, 113-125.

Yen, F., Wu, F., Tzeng, C., Lin, L., \& Ling, C. (2010). Curcumin nanoparticles improves the physicochemical properties of Curcumin and effectively enhances its antioxidant and antihepatoma activities. Journal of Agricultural and Food Chemistry, 58, 7376-7382

Yi, J., Fan, Y., Zhang, Y., Wen, Z., Zhao, L. \& Lu, Y. (2016). Glycosylated a-lactalbuminbased nanocomplex for curcumin: Physicochemical stability and DPPH-scavenging activity. Food Hydrocolloids, 61, 369-377.

Table 1. Bioactivity and cytotoxic properties of different curcumin colorant formulations. 


\begin{tabular}{|c|c|c|c|c|}
\hline & NC & DC & PC & Positive control \\
\hline \multicolumn{5}{|c|}{ Antioxidant activity $\left(\mathrm{EC}_{50}\right.$ values; $\left.\mu \mathrm{g} . \mathrm{mL}^{-1}\right)$} \\
\hline $\mathrm{DPPH}$ & $813 \pm 6^{b}$ & $1113 \pm 11^{\mathrm{a}}$ & $48 \pm 2^{c}$ & $41 \pm 1$ \\
\hline Reducing power & $552 \pm 5^{\mathrm{b}}$ & $858 \pm 4^{\mathrm{a}}$ & $46 \pm 1^{c}$ & $18 \pm 1$ \\
\hline$\beta$-carotene & $468 \pm 6^{b}$ & $691 \pm 17^{\mathrm{a}}$ & $41 \pm 3^{\mathrm{c}}$ & $42 \pm 1$ \\
\hline TBARS inhibition & $10.6 \pm 0.3^{b}$ & $19 \pm 1^{\mathrm{a}}$ & $7.9 \pm 0.1^{\mathrm{c}}$ & $23 \pm 1$ \\
\hline \multicolumn{5}{|c|}{ Anti-inflammatory activity $\left(\mathrm{EC}_{50}\right.$ values; $\left.\mu \mathrm{g} \cdot \mathrm{mL}^{-1}\right)$} \\
\hline Nitric oxide production & $202 \pm 5^{b}$ & $244 \pm 10^{\mathrm{a}}$ & $63 \pm 2^{c}$ & $16 \pm 1$ \\
\hline \multicolumn{5}{|c|}{ Cytotoxicity activity (GI50 values; $\mu \mathrm{g} \cdot \mathrm{mL}^{-1}$ ) } \\
\hline $\mathrm{MCF}-7$ & $47 \pm 5^{\mathrm{a}}$ & $47 \pm 2^{\mathrm{a}}$ & $29 \pm 3^{b}$ & $1.00 \pm 0.04$ \\
\hline NCI-H460 & $170 \pm 11^{\mathrm{a}}$ & $172 \pm 2^{\mathrm{a}}$ & $48 \pm 1^{\mathrm{b}}$ & $1.0 \pm 0.1$ \\
\hline HeLa & $90 \pm 2^{\mathrm{a}}$ & $75 \pm 1^{b}$ & $20 \pm 1^{\mathrm{c}}$ & $2.0 \pm 0.1$ \\
\hline HepG2 & $71 \pm 7^{b}$ & $153 \pm 18^{\mathrm{a}}$ & $17 \pm 1^{\mathrm{c}}$ & $1.0 \pm 0.2$ \\
\hline PLP2 & $206 \pm 11^{b}$ & $235 \pm 13^{\mathrm{a}}$ & $89 \pm 2^{c}$ & $3 \pm 1$ \\
\hline
\end{tabular}

Antibacterial activity (MIC; mg.mL $^{-1}$ )

\begin{tabular}{llcc}
\hline Gram positive & NC & DC & PC \\
\hline MRSA & 0.5 & 0.5 & 0.0625 \\
MSSA & 0.5 & 0.5 & 0.0625 \\
Enterococcus faecalis & 0.5 & 1 & 0.0625 \\
Listeria monocytogenes & 0.5 & 1 & 0.0625 \\
\hline Gram negative & & & \\
\hline Escherichia coli & 1 & 1 & 0.125 \\
Escherichia coli ESBL & 1 & 1 & 0.125 \\
Klebsiella pneumoniae & 1 & 1 & 0.125 \\
Klebsiella pneumoniae ESBL & 1 & 1 & 0.125 \\
Morganella morganii & 1 & - & 0.5 \\
Pseudomonas aeruginosa & 1 & - & 0.5
\end{tabular}

*Trolox, dexamethasone and ellipticine were used as positive controls for antioxidant, anti-inflammatory and antitumor and hepatotoxicity activities, respectively. In each column, different letters mean significant statistical differences between the different formulations of curcumin $(\mathrm{p}<0.05) . \mathrm{MIC}=$ minimal inhibition concentration; $\mathrm{ESBL}=$ spectrum extended producer of $\beta$-lactamases; MRSA = methicillin-resistant Staphylococcus aureus; MSSA = methicillin-sensitive Staphylococcus aureus. Curcumin powder: PC; water-dispersible curcumin: DC; and nanoencapsulated curcumin: NC. 
Table 2. Nutritional composition (g.100 g $\mathrm{g}^{-1}$ fresh yogurt), individual sugars energy (g.100 g $\mathrm{g}^{-1}$ fresh yogurt) and energy values (kcal.100 $\mathrm{g}^{-1}$ fresh yogurt) for different yogurt types (YF) and storage times (ST). Results are presented as mean \pm standard deviation. ${ }^{1}$

\begin{tabular}{|c|c|c|c|c|c|c|c|c|c|}
\hline & & Water & Fat & Protein & Ash & Carbohydrates & Galactose & Lactose & Energy \\
\hline \multirow{5}{*}{ YF } & $\mathrm{YC}$ & $83 \pm 1$ & $3.6 \pm 0.2^{\mathrm{bc}}$ & $5.2 \pm 0.1$ & $0.76 \pm 0.05$ & $6.2 \pm 0.2$ & $0.91 \pm 0.04$ & $3.9 \pm 0.1$ & $78 \pm 2$ \\
\hline & YPC & $84 \pm 1$ & $3.5 \pm 0.1^{\mathrm{c}}$ & $5.1 \pm 0.2$ & $0.79 \pm 0.05$ & $6.0 \pm 0.3$ & $1.03 \pm 0.03$ & $4.1 \pm 0.1$ & $77 \pm 1$ \\
\hline & YDC & $83 \pm 1$ & $3.8 \pm 0.1^{\mathrm{a}}$ & $5.3 \pm 0.2$ & $0.81 \pm 0.02$ & $6.0 \pm 0.1$ & $0.90 \pm 0.04$ & $3.9 \pm 0.1$ & $79 \pm 1$ \\
\hline & YNC & $84 \pm 1$ & $3.7 \pm 0.1^{\mathrm{b}}$ & $5.4 \pm 0.3$ & $0.80 \pm 0.02$ & $5.9 \pm 0.3$ & $0.88 \pm 0.05$ & $3.8 \pm 0.1$ & $78 \pm 2$ \\
\hline & $\overline{p \text {-value }(\mathrm{n}=27)^{2}}$ & $<0.001$ & $<0.001$ & $<0.001$ & $<0.001$ & 0.002 & $<0.001$ & $<0.001$ & $<0.001$ \\
\hline \multirow{4}{*}{ ST } & 0 days & $84 \pm 1$ & $3.7 \pm 0.2$ & $5.1 \pm 0.1$ & $0.43 \pm 0.05$ & $6.0 \pm 0.3$ & $0.90 \pm 0.05$ & $3.9 \pm 0.2$ & $78 \pm 2$ \\
\hline & 7 days & $82 \pm 1$ & $3.6 \pm 0.1$ & $5.4 \pm 0.3$ & $0.42 \pm 0.05$ & $6.2 \pm 0.2$ & $0.93 \pm 0.05$ & $4.0 \pm 0.2$ & $79 \pm 1$ \\
\hline & 15 days & $84 \pm 1$ & $3.7 \pm 0.1$ & $5.2 \pm 0.2$ & $0.36 \pm 0.04$ & $5.9 \pm 0.2$ & $0.96 \pm 0.05$ & $3.9 \pm 0.1$ & $77 \pm 2$ \\
\hline & $\overline{p \text {-value }(\mathrm{n}=36)^{3}}$ & $<0.001$ & 0.058 & $<0.001$ & $<0.001$ & $<0.001$ & $<0.001$ & 0.068 & 0.006 \\
\hline$\overline{\mathrm{YF} \times \mathrm{ST}}$ & $p$-value $(\mathrm{n}=108)^{4}$ & $<0.001$ & 0.394 & $<0.001$ & $<0.001$ & $<0.001$ & $<0.001$ & $<0.001$ & $<0.001$ \\
\hline
\end{tabular}

${ }^{1}$ Results are reported as mean values of each YF, including results from 0,3 and 7 days, and mean values of each ST, considering all YF. ${ }^{2}$ If $p<0.05$, the corresponding parameter presented a significantly different value for at least one YF (identified with different letters). ${ }^{3}$ If $p<0.05$, the corresponding parameter presented a significantly different value for at least one ST. ${ }^{4}$ For values above 0.05 , the interaction was considered as non-significant. YC: control yogurt; YPC: yogurts incorporated with curcumin powder; YDC: yogurts incorporated with commercial water-dispersible formulation; and YNC: yogurts incorporated with the produced nanoparticles formulation. 
Table 3. Color parameters for different yogurt types (YF) and storage times (ST). Results are presented as mean \pm standard deviation. ${ }^{1}$

\begin{tabular}{llccc}
\hline & & $L^{*}$ & $a^{*}$ & $b^{*}$ \\
\hline \multirow{4}{*}{ YF } & YC & $93 \pm 1$ & $-3.4 \pm 0.1$ & $10 \pm 1$ \\
& YPC & $88 \pm 2$ & $0 \pm 2$ & $32 \pm 6$ \\
& YDC & $93 \pm 1$ & $-8 \pm 1$ & $21 \pm 1$ \\
& YNC & $93 \pm 1$ & $-8 \pm 2$ & $27 \pm 1$ \\
\cline { 2 - 5 } & $p$-value $(\mathrm{n}=27)^{2}$ & $<0.001$ & $<0.001$ & $<0.001$ \\
\hline & & & & $20 \pm 6$ \\
& & $92 \pm 3$ & $-3 \pm 4$ & $23 \pm 9$ \\
ST & 7 days & $92 \pm 2$ & $-6 \pm 3$ & $24 \pm 10$ \\
& 15 days & $92 \pm 2$ & $-6 \pm 3$ & 0.149 \\
\cline { 2 - 5 } & $p$-value $(\mathrm{n}=36)^{3}$ & 0.263 & 0.014 & $<0.001$ \\
\hline YF $\times$ ST & $p$-value $(\mathrm{n}=108)^{4}$ & $<0.001$ & $<0.001$ &
\end{tabular}

${ }^{1}$ Results are reported as mean values of each YF, including results from 0,3 and 7 days, and mean values of each $\mathrm{ST}$, considering all $\mathrm{YF} .{ }^{2} \mathrm{If} p<0.05$, the corresponding parameter presented a significantly different value for at least one YF. ${ }^{3}$ If $p<0.05$, the corresponding parameter presented a significantly different value for at least one ST. ${ }^{4}$ In this table, the interaction among factors was significant in all cases; thereby no multiple comparisons could be performed. YC: control yogurt; YPC: yogurts incorporated with curcumin powder; YDC: yogurts incorporated with commercial water-dispersible formulation; and YNC: yogurts incorporated with the produced nanoparticles formulation. 
Table 4. Major (above 1\%) fatty acids profile (relative percentages) for different yogurt types (YF) and storage times (ST). Results are presented as mean \pm standard deviation. ${ }^{1}$

\begin{tabular}{|c|c|c|c|c|c|c|c|c|c|c|c|c|}
\hline & & C6:0 & C10:0 & C12:0 & C14:0 & C16:0 & C18:0 & C18:1n9c & C18:2n6c & SFA & MUFA & PUFA \\
\hline \multirow{5}{*}{ YF } & $\mathrm{YC}$ & $3.2 \pm 0.3$ & $3.7 \pm 0.1$ & $4.2 \pm 0.1$ & $12.7 \pm 0.1$ & $35 \pm 1$ & $10.3 \pm 0.2$ & $20.6 \pm 0.3$ & $2.1 \pm 0.2$ & $74 \pm 1$ & $22.7 \pm 0.4$ & $3.4 \pm 0.2$ \\
\hline & YPC & $3.2 \pm 0.4$ & $3.8 \pm 0.2$ & $4.3 \pm 0.1$ & $12.9 \pm 0.3$ & $35 \pm 1$ & $10.3 \pm 0.3$ & $20.1 \pm 0.5$ & $2.0 \pm 0.3$ & $75 \pm 1$ & $22.2 \pm 0.5$ & $3.2 \pm 0.5$ \\
\hline & YDC & $3.7 \pm 0.5$ & $3.9 \pm 0.4$ & $4.3 \pm 0.1$ & $12.6 \pm 0.3$ & $35 \pm 1$ & $10.2 \pm 0.3$ & $20.2 \pm 0.3$ & $2.2 \pm 0.3$ & $74 \pm 1$ & $22.3 \pm 0.3$ & $3.5 \pm 0.3$ \\
\hline & YNC & $3.2 \pm 0.4$ & $3.7 \pm 0.2$ & $4.2 \pm 0.1$ & $12.7 \pm 0.2$ & $35 \pm 1$ & $10.3 \pm 0.2$ & $20.4 \pm 0.4$ & $2.1 \pm 0.1$ & $74 \pm 1$ & $22.5 \pm 0.3$ & $3.4 \pm 0.2$ \\
\hline & $\overline{p \text {-value }(\mathrm{n}=27)^{2}}$ & $<0.001$ & 0.002 & $<0.001$ & $<0.001$ & 0.001 & 0.187 & $<0.001$ & 0.010 & $<0.001$ & $<0.001$ & 0.005 \\
\hline \multirow{4}{*}{ ST } & 0 days & $3.2 \pm 0.3$ & $3.6 \pm 0.2$ & $4.2 \pm 0.1$ & $12.7 \pm 0.1$ & $36 \pm 1$ & $10.4 \pm 0.2$ & $20.4 \pm 0.5$ & $2.0 \pm 0.2$ & $74 \pm 1$ & $22.4 \pm 0.5$ & $3.2 \pm 0.4$ \\
\hline & 7 days & $3.2 \pm 0.4$ & $3.7 \pm 0.2$ & $4.3 \pm 0.1$ & $12.9 \pm 0.3$ & $35 \pm 1$ & $10.3 \pm 0.3$ & $20.3 \pm 0.3$ & $2.2 \pm 0.3$ & $74 \pm 1$ & $22.4 \pm 0.3$ & $3.4 \pm 0.3$ \\
\hline & 15 days & $3.6 \pm 0.5$ & $4.0 \pm 0.4$ & $4.3 \pm 0.1$ & $12.6 \pm 0.3$ & $34 \pm 1$ & $10.2 \pm 0.3$ & $20.3 \pm 0.4$ & $2.2 \pm 0.1$ & $74 \pm 1$ & $22.5 \pm 0.4$ & $3.6 \pm 0.2$ \\
\hline & $p$-value $(\mathrm{n}=36)^{3}$ & $<0.001$ & $<0.001$ & 0.005 & $<0.001$ & $<0.001$ & 0.001 & 0.372 & 0.001 & 0.124 & 0.307 & $<0.001$ \\
\hline $\mathrm{YF} \times \mathrm{ST}$ & $p$-value $(\mathrm{n}=108)^{4}$ & $<0.001$ & $<0.001$ & $<0.001$ & $<0.001$ & $<0.001$ & $<0.001$ & $<0.001$ & $<0.001$ & $<0.001$ & $<0.001$ & $<0.001$ \\
\hline
\end{tabular}

${ }^{1}$ Results are reported as mean values of each YF, including results from 0,3 and 7 days, and mean values of each ST, considering all YF. ${ }^{2}$ If $p<0.05$, the corresponding parameter presented a significantly different value for at least one YF. ${ }^{3}$ If $p<0.05$, the corresponding parameter presented a significantly different value for at least one ST. ${ }^{4}$ In this table, the interaction among factors was significant in all cases; thereby no multiple comparisons could be performed. Caproic acid (C6:0); capric acid (C10:0); lauric acid (C12:0); myristic acid (C14:0); palmitic acid (C16:0); stearic acid (C18:0); oleic acid (C18:1n9); linoleic acid (C18:2n6c); SFA - saturated fatty acids; MUFA - monounsaturated fatty acids; PUFA - polyunsaturated fatty acids. YC: control yogurt; YPC: yogurts incorporated with curcumin powder; YDC: yogurts incorporated with commercial water-dispersible formulation; and YNC: yogurts incorporated with the produced nanoparticles formulation. 

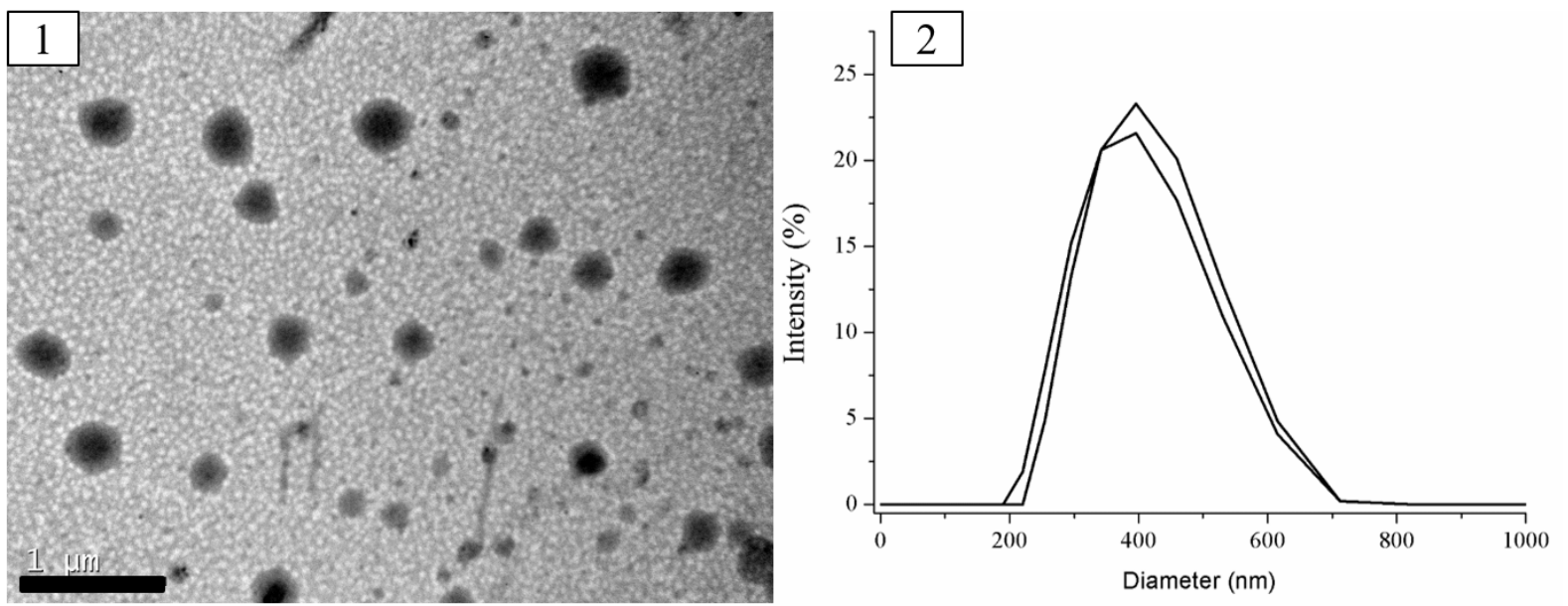

A
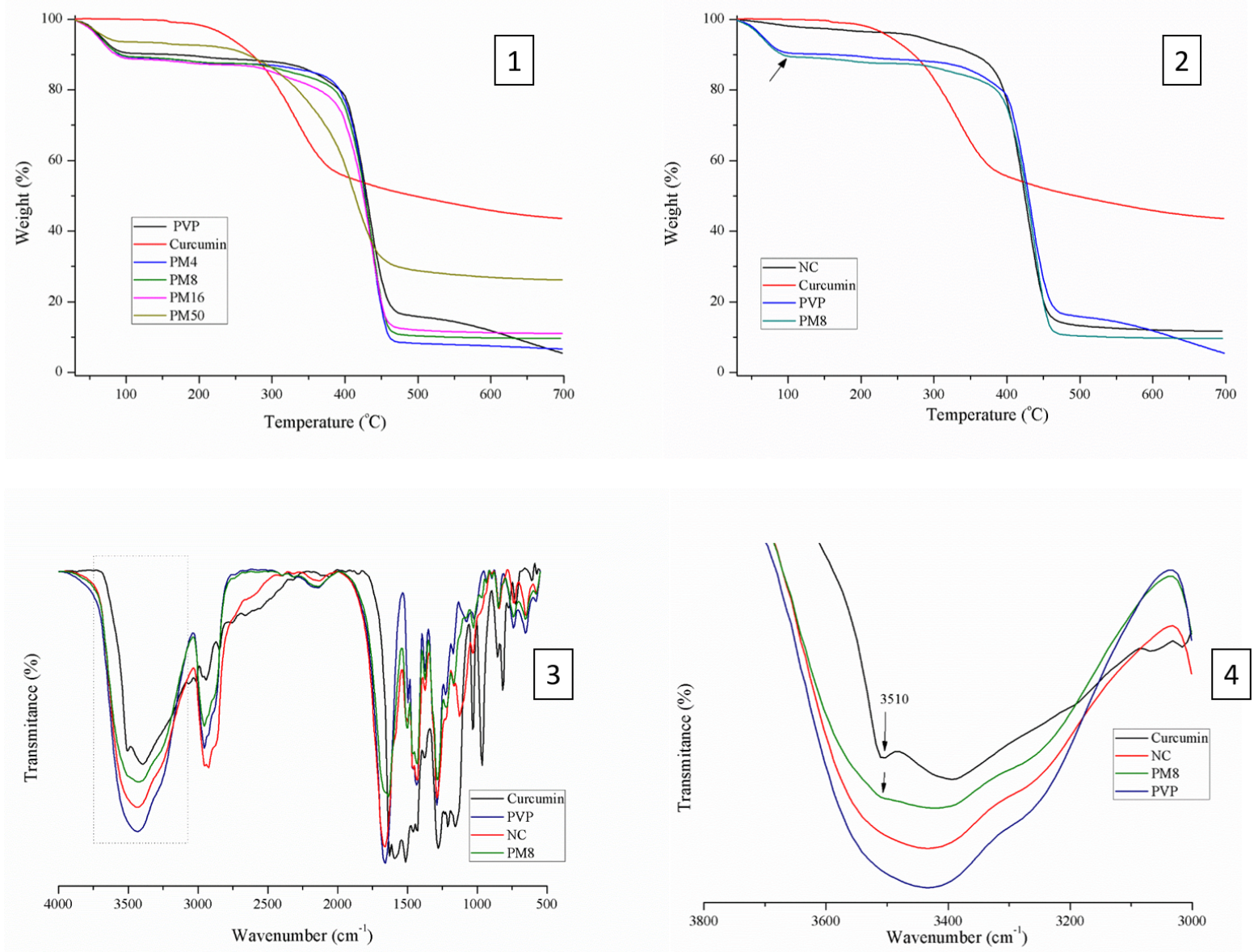

$\mathrm{B}$

FIGURE 1. (A) PVP-CURCUMIN NANOPARTICLES SIZE AND MORPHOLOGICAL CHARACTERIZATION: 1) TEM IMAGE (SIZE BAR $=1 \mu \mathrm{M})$; 2) DLS INTENSITY SIZE DISTRIBUTION (DUPLICATE). (B) THERMOGRAVIMETRIC CURVES ( 1 - PVP, CURCUMIN AND PHYSICAL MIXTURES OF PVP WITH CURCUMIN AT 4\%WT: PM4, 8\%WT: PM8, 16\%WT: PM16 AND 50\%WT: PM50; 2 - NANOENCAPSULATED CURCUMIN: NC, CURCUMIN, PVP AND 
PM8) AND FTIR SPECTRA OF CURCUMIN, PVP, NC AND PM8 (3 - COMPLETE SPECTRA; 4 - CURCUMIN OH STRETCHING BAND).

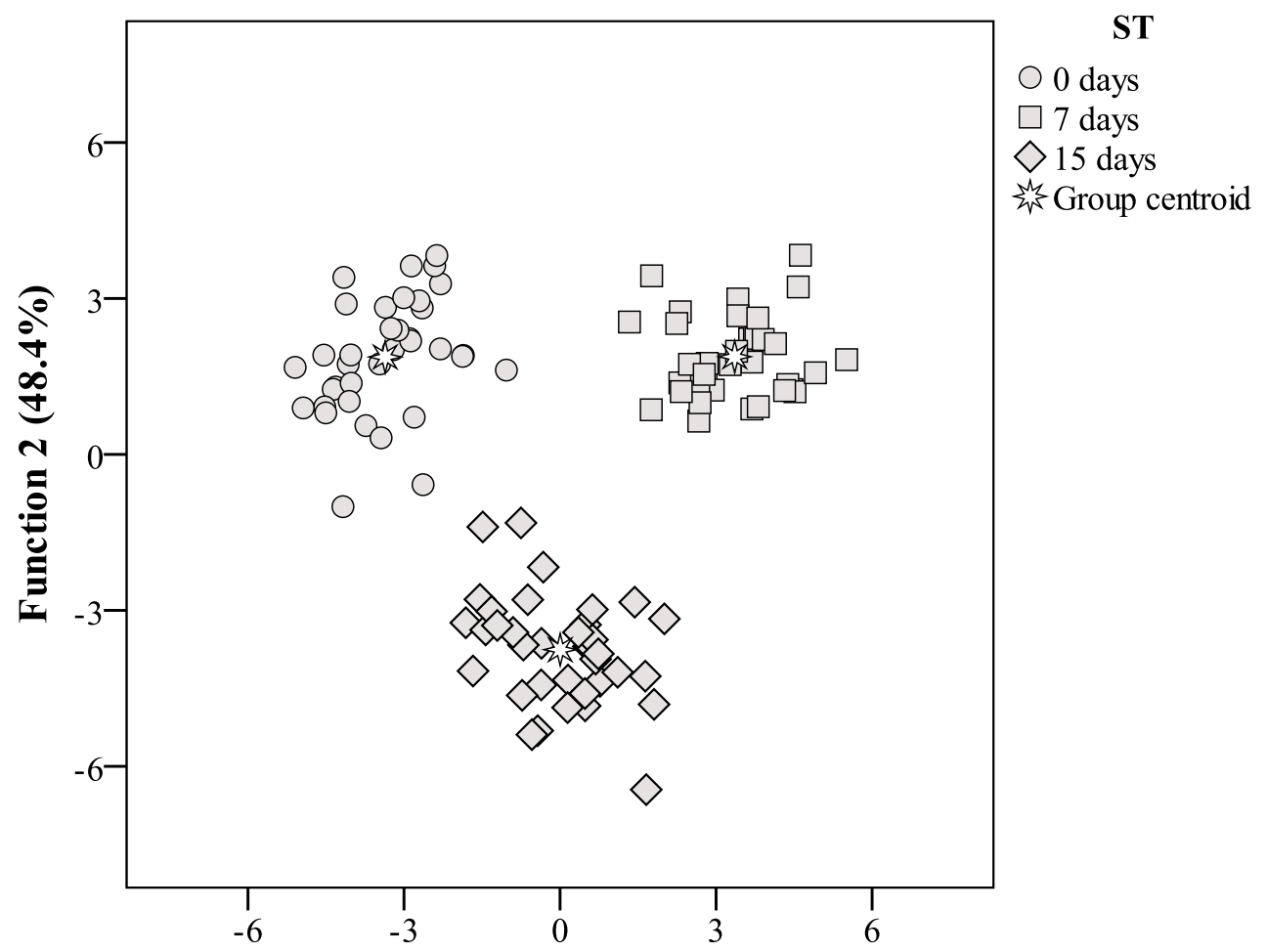

Function 1 (51.6\%)

(A)

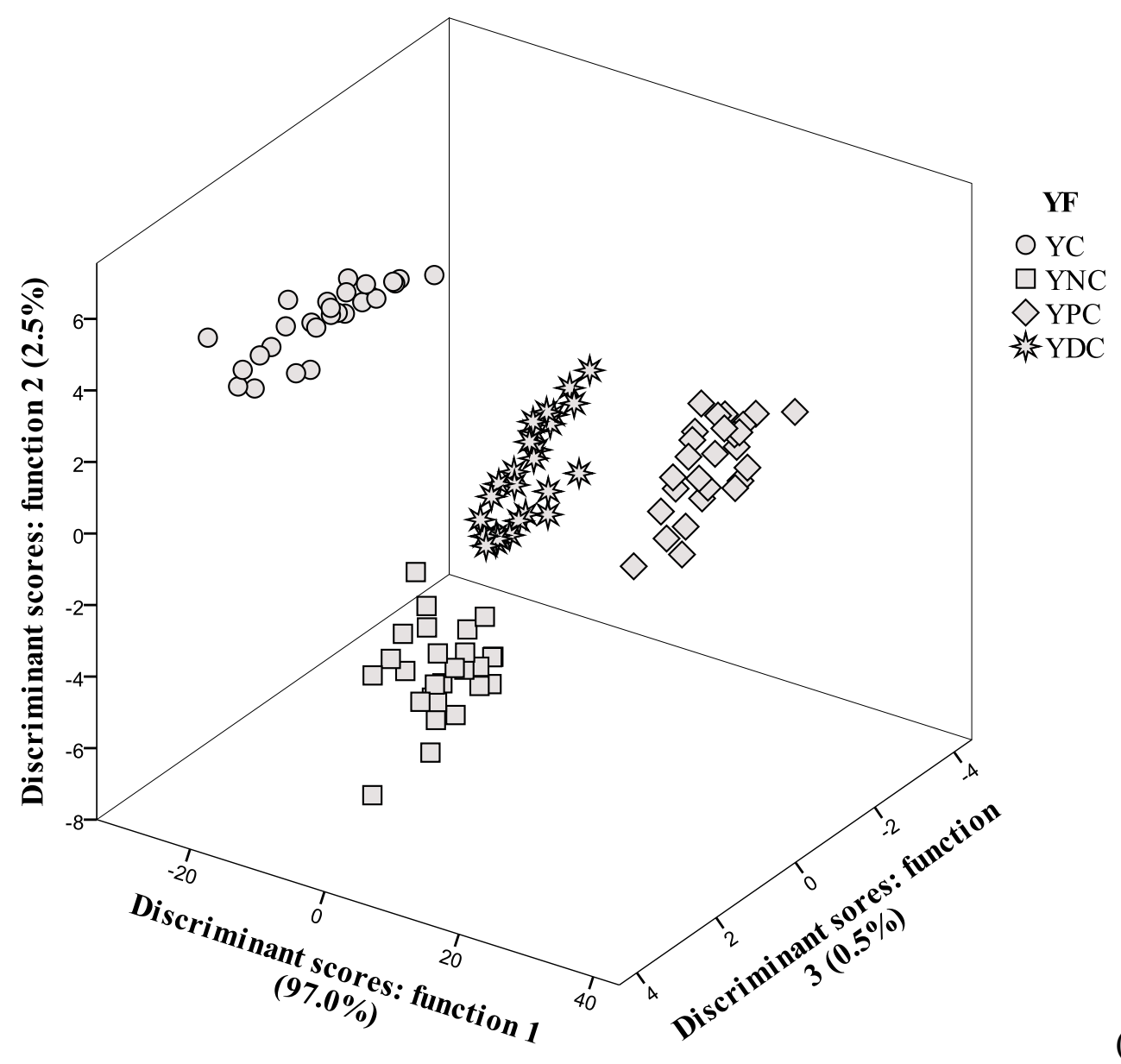

(B) 
Figure 2. Spatial distribution of ST (A) and YF (B) markers distributed by the canonical discriminant functions coefficients defined according to the results obtained in the laboratorial assays. 